\title{
Seroprevalence of hepatitis B virus in Taiwan 30 years after the commencement of the national vaccination program
}

\author{
Yang-Cheng Hu ${ }^{1}$, Chih-Ching Yeh ${ }^{1,2}$, Ruey-Yu Chen ${ }^{1}$, Chien-Tien Su ${ }^{1,3}$ ， Wen-Chang Wang ${ }^{4}$, Chyi-Huey Bai \\ 1,5 , Chi-Fei Chan ${ }^{6}$, Fu Hsiung Su Corresp. 5, 7, 8, 9 \\ ${ }^{1}$ School of Public Health, College of Public Health, Taipei Medical University, Taipei City, Taiwan \\ 2 Department of Public Health, China Medical University,, Taichung City, Taiwan \\ 3 Department of Family Medicine, Taipei Medical University Hospital, Taipei City, Taiwan \\ 4 The Ph.D. Program for Translational Medicine, College of Medical Science and Technology, Taipei Medical University, Taipei City, Taiwan \\ 5 Department of Public Health, School of Medicine, College of Medicine, Taipei Medical University, Taipei City, Taiwan \\ 6 Fu Jen Clinic, College of Medicine, Fu Jen Catholic University, New Taipei City, Taiwan \\ 7 School of Medicine, College of Medicine, Fu Jen Catholic University, New Taipei City, Taiwan \\ 8 Division of Family Medicine, Department of Community Medicine and Long Term Care, Fu Jen Catholic University Hospital, New Taipei City, Taiwan \\ 9 Department of Family Medicine, Wan Fang Hospital, Taipei Medical University, Taipei City, Taiwan \\ Corresponding Author: Fu Hsiung Su \\ Email address: williamsufh1@yahoo.com.tw
}

\section{Background}

In this study, the long-term efficacy of hepatitis B virus (HBV) vaccination was assessed using seroprevalence and an age-period-cohort (APC) model of HBV seromarkers among university entrants 30 years after the introduction of the national neonatal HBV vaccination program in Taiwan.

\section{Methods}

In total, data of 17,611 university entrants who underwent university entrance health examinations between 2005 and 2016 were included. The seroprevalence of the HBV surface antigen (HBsAg) and the levels of the antibody against the HBV surface antigen (anti-HBs) in each year group and sex were calculated. The levels of the antibody against the HBV core antigen were examined only for 2012 and 2016. The APC model was used to analyze the HBV carrier rates.

\section{Results}

The chronic HBV infection (HBsAg positivity) rate decreased from 9.7\% in university students born before June 1974 to $<1.0 \%$ in students born after 1992. The prevalence of anti-HBs positivity declined, particularly between the 1984-1988 cohort (78.2\%-53.2\%) and the 1990-1994 cohort (60.6\%-44.4\%). Our APC model revealed that the chronic HBV carrier rate among the student population was affected significantly by age, period, and cohort $(P<0.001)$.

\section{Conclusions}

HBV vaccination is one of the most effective strategies for preventing HBV infection. However, for complete eradication of HBV infection, the development of strategies that detect vaccination failure more effectively than current strategies do and early implementation of appropriate treatments are both necessary. 
1 Seroprevalence of Hepatitis $B$ Virus in Taiwan 30 years after the 2 Commencement of the National Vaccination Program

3

4 Yang-Cheng $\mathrm{Hu}^{\mathrm{a},+}$, Chih-Ching Yeh ${ }^{\mathrm{a}, \mathrm{b},+}$, , Ruey-Yu Chen ${ }^{\mathrm{a}}$, Chien-Tien Su ${ }^{\mathrm{a}, \mathrm{c}}$, Wen-Chang Wang

5 d, Chyi-Huey Bai a,e, Chi-Fei Chan ${ }^{\mathrm{f}}$, Fu-Hsiung Su g, h,i,j*

7 a School of Public Health, College of Public Health, Taipei Medical University, Taipei, Taiwan

8 b Department of Public Health, China Medical University, Taichung, Taiwan

$9 \quad{ }^{\mathrm{c}}$ Department of Family Medicine, Taipei Medical University Hospital, Taipei, Taiwan

$10 \mathrm{~d}$ The Ph.D. Program for Translational Medicine, College of Medical Science and Technology, 11 Taipei Medical University, Taipei, Taiwan

12 e Department of Public Health, School of Medicine, College of Medicine, Taipei Medical 13 University, Taipei, Taiwan

$14{ }^{\mathrm{f}} \mathrm{Fu}$ Jen University Clinic, Fu Jen Catholic University, New Taipei City, Taiwan

$15{ }^{\mathrm{g}}$ School of Medicine, College of Medicine, Fu Jen Catholic University, New Taipei City, Taiwan $16{ }^{\mathrm{h}}$ Division of Family Medicine, Department of Community Medicine and Long Term Care, Fu Jen

17 Catholic University Hospital, New Taipei City, Taiwan

18 i Department of Public Health, School of Medicine, College of Medicine, Taipei Medical 19 University, Taipei, Taiwan

20 j Department of Family Medicine, Wan Fang Hospital, Taipei Medical University, Taipei, Taiwan 21

22

23

$24+$ : contributed equally

25

26 Corresponding author:

$27 \quad$ Fu-Hsiung Su

28 EMAIL: williamsufh1@yahoo.com.tw 
30 ABSTRACT

\section{Background}

32 In this study, the long-term efficacy of hepatitis B virus (HBV) vaccination was assessed using 33 seroprevalence and an age-period-cohort (APC) model of HBV seromarkers among university 34 entrants 30 years after the introduction of the national neonatal HBV vaccination program in 35 Taiwan.

\section{Methods}

37 In total, data of 17,611 university entrants who underwent university entrance health examinations 38 between 2005 and 2016 were included. The seroprevalence of the HBV surface antigen (HBsAg) 39 and the levels of the antibody against the HBV surface antigen (anti-HBs) in each year group and 40 sex were calculated. The levels of the antibody against the HBV core antigen were examined only 41 for 2012 and 2016. The APC model was used to analyze the HBV carrier rates.

\section{Results}

43 The chronic HBV infection (HBsAg positivity) rate decreased from 9.7\% in university students 44 born before June 1974 to $<1.0 \%$ in students born after 1992. The prevalence of anti-HBs positivity 45 declined, particularly between the 1984-1988 cohort (78.2\%-53.2\%) and the 1990-1994 cohort $46(60.6 \%-44.4 \%)$. Our APC model revealed that the chronic HBV carrier rate among the student 47 population was affected significantly by age, period, and cohort $(P<0.001)$.

\section{Conclusions}

49 HBV vaccination is one of the most effective strategies for preventing HBV infection. However, 50 for complete eradication of HBV infection, the development of strategies that detect vaccination 51 failure more effectively than current strategies do and early implementation of appropriate 52 treatments are both necessary. 


\section{Introduction}

56

57 Worldwide, hepatitis B virus (HBV) infection is a major cause of chronic hepatitis, liver

58 cirrhosis, and hepatocellular carcinomas (Beasley \& Hwang 1984), and it continues to contribute

59 to the most serious challenges currently posed by infectious diseases in public health. Although

60 some countries, such as Taiwan, already have high immunization coverage, more than 350 million

61 people worldwide have chronic HBV infection, with the majority of the infected people living in

62 Africa and Asia (WHO 2017). HBV and related complications result in nearly 600,000 deaths

63 annually (Perz et al. 2006); therefore, despite the high vaccination coverage in many countries,

64 HBV prevalence remains a major public health burden.

65

Prior to the introduction of the national HBV vaccination program in Taiwan in 1984, approximately $15 \%-20 \%$ of Taiwanese adults tested positive for the HBV surface antigen

67 (HBsAg), with mother-infant vertical transmission being the primary means of infection (Gust 1996; Sung 1984). The nationwide HBV vaccination program was officially implemented in July 1984 in Taiwan. During the first 2 years of the program, the vaccination was available free-of-

70 charge only to infants born to HBsAg-carrier mothers. A four-dose plasma-derived vaccine

71 regimen was provided; the doses were administered at birth and at 1, 2, and 12 months of age

72 (Chen et al. 1987). However, from July 1986 onwards, all infants were immunized against HBV

73 by using the four-dose plasma-derived vaccine. Neonates born to highly infectious carrier mothers 
74 also received $0.5 \mathrm{~mL}$ of $\mathrm{HBV}$ immunoglobulin at birth. In addition, after November 1, 1992, the

75 plasma-derived vaccine used for HBV vaccination was replaced by a recombinant yeast-derived

76 vaccination with a three-dose regimen; the doses were administered at birth and at the ages of 1

77 and 6 months. From October 1990, the free catch-up HBV vaccination program was extended to

78 include all children aged $<7$ years, all involved medical personnel, and selected groups of children

79 (e.g., elementary-school children in aboriginal areas and offshore islands). The details of the

80 program have been extensively documented previously (Su et al. 2008).

81 The program was highly successful, and within 12 years of its implementation, over 20

82 million vaccinations are estimated to have been provided to neonates, children, and secondary

83 school and college students. Approximately $89 \%$ of the 3.2 million vaccinated infants completed

84 their three-dose recombinant vaccination regimen, and at least $90 \%$ of the children currently aged

$85<15$ years have received the vaccination (Ni et al. 2001). Additional statistics from the Ministry of

86 Health and Welfare, Taiwan, revealed that the 2016 newborn HBV vaccination rate was as high

87 as $97.8 \%$, which is the highest among the standard vaccines listed for infants (CDC 2017). The

88 program's effectiveness has been further demonstrated by numerous studies that have reported a

89 decrease in vital HBV prevalence markers, namely overall HBsAg-carrier rate, chronic HBV

90 infection incidence rate, and mother-to-child transmission infection rate, since the commencement

91 of the vaccination program (Ni et al. 2016; Ni et al. 2012; Ni et al. 2007; Su et al. 2007a; Su et al. 
92 2007b; Tsen et al. 1991).

93 Many studies have reported that neonatal HBV vaccination provides sufficient protection

94 against $\mathrm{HBV}$ infections and that disease prevalence is significantly lower in the postvaccination

95 generation than in the prevaccination generation (Chien et al. 2006; Ni et al. 2016; Ni et al. 2012;

96 Ni et al. 2007; Su et al. 2007a; Su et al. 2007b). However, previous studies have primarily used

97 unadjusted age for analysis and the cohorts and have rarely examined age, period, and cohort

98 effects. According to our review of relevant studies, although HBV prevalence in Taiwan generally

99 increases with age, this phenomenon becomes less apparent if the populations before and after

100 vaccination are exclusively and independently observed, demonstrating that disease prevalence is

101 likely to be affected by different time variables (Chien et al. 2006; Ni et al. 2016; Ni et al. 2012;

$102 \mathrm{Ni}$ et al. 2007). Consequently, period and cohort effects should be considered when analyzing the

103 relationship between age, vaccination, and disease.

104 Therefore, in this study, we examined whether age-period-cohort effects were significantly

105 associated with HBV prevalence and determined whether disease prevalence was maintained

106 below a threshold level after 30 years of universal neonatal HBV vaccination program

107 implementation. Although the universal neonatal HBV vaccination program proved effective in

108 both immunization coverage and disease prevention, continuous disease monitoring is crucial for

109 disease control. 


\section{Material and Methods}

111

112

113

114

115

116

117

118

119

120

121

122

123

124

125

126

127

\subsection{Data collection and delinking}

We collected data from the records of entrance health examinations of medical university students from 2005 to 2016 in northern Taiwan. The records contained data of undergraduate and graduate entrants. All data collected for this study were delinked by the university's office of environmental protection and occupational safety. However, demographic variables such as sex, the first letter of the social identification number, birth date, and serological markers of HBV, including seropositivity for HBsAg, HBV surface antibody (anti-HBs), and HBV core antibody (anti-HBc), were retained. Serum anti-HBc titer data were available only for 2012 and 2016. However, before delinking, the office screened and eliminated the duplicate records of undergraduates who completed their graduate studies in the same institution and retained only their initial undergraduate entrance records. Because the data collected for this study were delinked by the university's office of environmental protection and occupational safety, our study was approved, and the requirement of student informed consent was waived by the Joint Institutional Review Board of Taipei Medical University (N201603069).

\subsection{Seromarkers for $\mathrm{HBV}$ detection}

The serum levels of HBsAg, anti-HBs, and anti-HBc were determined using a commercially available enzyme immunoassay kit (Elecsys 2010 system, Roche Diagnostics, Mannheim, 
128 Germany)(GmbH BM 1998; GmbH RD 2007a; GmbH RD 2007b). The detection limit of the anti-

129 HBs enzyme immunoassay kit was $0.1 \mathrm{mIU} / \mathrm{mL}$. Samples with an anti-HBs titer of $\geq 10.0 \mathrm{mIU} / \mathrm{mL}$

130 were interpreted as protective. Sample rate/cutoff rate $(\mathrm{S} / \mathrm{CO})$ for anti-HBc of $\leq 1.000$ were

131 considered to represent reactivity to anti-HBc, and values between 1.001 and 3.000 were

132 considered negative reactions. $\mathrm{S} / \mathrm{CO}$ of $\mathrm{HBsAg} \geq 1.00$ were considered to represent $\mathrm{HBsAg}$

133 reactivity, and samples with $\mathrm{HBsAg}<1.00$ were considered negative reactions.

134

135

136

138

139

140

141 after November 1, 1992 (who received the recombinant HBV vaccine). Cohorts C and D

142 represented the period during which the vaccination program was extended to all newborns from

143 July 1, 1986. Moreover, the students were grouped according to age. Periods were classified

144 according to the entry year of the participants; accordingly, our model consisted of 12 periods from

1452005 to 2016. 
146

147

148

149

150

151

152

153

154

155

156

157

158

159

160

161

162 163

\section{Statistical analysis}

The chi-squared $\left(\chi^{2}\right)$ test was used for analyzing categorical variables, and descriptive data were presented as mean \pm standard deviation. The SPSS 19.0 software package (Chicago, IL, USA) was used for data analysis, and $\alpha$ values $<0.05$ were considered statistically significant.

The age-period-cohort (APC) model was used to analyze the HBsAg status assuming that the model fit the Poisson regression distribution. A log-linear Poisson regression model was used for analysis:

Formula: 1 o $g\left(\frac{d_{i j}}{y_{i_{j} j}}\right)=\mu+\alpha_{i}+\beta_{j}+\gamma_{k}+\varepsilon$ where (dij/yij): the rate of interest, dij: the number of cases in the $i$ th age group and $j$ th period, yij: populations in the $i$ th age group and $j$ th period that are at risk, $\alpha \mathrm{i}$ : the effect of the $i$ th age group,

$\beta \mathrm{j}:$ the effect of the $j$ th period, and $\gamma \mathrm{k}$ : the effect of the $k$ th cohort.

The deviance and degrees of freedom were the factors used in the goodness-of-fit test.

Variables were added to the age-period, age-cohort, and age-period-cohort models to analyze whether the results were altered; an alteration would imply that the factors affected the models. The deviance and degree of freedom were varied to determine whether the significance of the 
164 estimated $\chi^{2}$ value was changed for evaluation purpose.

\section{2. Results}

166

167

168

169

170

171

172

173

174

175

176

177

178

179

180

181

\subsection{Seroprevalence of $\mathrm{HBV}$}

Originally, a total of 18,783 students were selected for this study. Twenty students whose records lacked demographic data, 522 international students, and 630 students with incomplete data on HBV seromarkers were excluded. Finally, 17,611 students finally were included in the analysis. To analyze the seroprevalence of HBV, this study included the HBsAg and anti-HBs test results of students who had enrolled between 2005 and 2016. The chronic HBV infection (HBsAg positivity) rate decreased from $9.7 \%$ in university students born before June 1974 to $<1 \%$ in students born after July 1992 (Table 1). The overall chronic HBV infection rate decreased considerably from 1979 to 1988 and has stayed below 1\% since 1992 (Figure 1). In our cohort, the rate of occurrence of chronic HBV carriers were higher in the male students than in the female students.

The proportions of HBV immunity due to hepatitis B vaccination or natural infection (HBsAg negative and anti-HBs positive cases) were calculated in each birth year group (Table 1). Two stages of decline were observed, first between the 1984-1986 (78.2\%) and 1986-1988 (53.2\%) cohorts and then between the 1990-1992 (60.6\%) and 1992-1994 (44.4\%) cohorts. The proportions were stable throughout the rest of the timeline, thus producing a stepwise pattern of 
182 change (Figure 2). Female students tended to preserve higher HBV immunity (anti-HBs positivity)

183 rates than their male counterparts did.

184 According to our 2008 study, the prevalence of positive serum anti- $\mathrm{HBc}$ among the students in

185 birth cohort years 1976,1984 , and 1986 were $48.7 \%, 9.8 \%$ and $4.0 \%$, respectively. The prevalence

186 of HBV immunity due to natural infection (HBsAg negative, anti-HBc positive, and anti-HBs

187 positive) was $32.9 \%, 6.8 \%$, and $2.1 \%$, respectively ( $\mathrm{Su}$ et al. 2008). The total prevalence rate of

188 anti-HBc positivity in students aged $18-20$ years was $4.3 \%$ in 2008. In this study, we also included

189 the serum data of anti-HBc levels among students aged 18-20 years in 2012 and 2016, the mean

190 values of which were $1.7 \%$ and $0.6 \%$, respectively. The prevalence rates of HBV immunity due to

191 natural infection were $0.5 \%$ and $0.6 \%$, respectively, in 2012 and 2016.

192

\subsection{Age-period-cohort model}

193

Table 2 presents the population distribution of the different birth cohorts. A total of 17,611

194 students were included in this study. A large proportion of the students belonged to cohort C, which constituted $41.0 \%$ of the study population. Cohort B had the fewest (968) students. The average age of the 17,611 students was $22.6 \pm 6.8$ years. Cohort A, with an average age of $32.8 \pm$ 7.8 years, was significantly older than the other cohorts $(P<0.001)$ and had the largest age range:

21.1-70.5 years, whereas cohort D was the youngest, with an average age of $18.9 \pm 1.0$ years and 
200 the study population, but their distribution differed significantly among the cohorts $(P<0.001)$. If

201 grouped according to their enrollment years, estimated 1,324-1,611 cases were tested each year.

202 No case before 2010 was assigned to cohort D.

203 The APC model was developed to illustrate the relationship between temporal variables and

204 chronic HBV infection (serum HBsAg positivity) among the student population enrolled between

2052005 and 2016 in a medical university in northern Taiwan. Table 3 shows the deviance and

206 likelihood ratio test results of age, period, cohort, and their combination effects on the APC

207 models. Among these models, the age, period, and cohort effects were significant at the 5\% level

208 (all $P<0.001$ ). However, magnitude of the age effect was the highest (deviance difference $=492.7$,

$209 \mathrm{df}=9$ ) when the age + period + cohort effect was the reference. The cohort effect was also highly

210 significant (deviance difference $=287.7, \mathrm{df}=6$ ). Therefore, the magnitude of the age + cohort

211 effect was the highest (deviance difference $=219.6, \mathrm{df}=2$ ) among the age-period, period-cohort,

212 and age-cohort models.

213

214 Discussion

215 The results of this study suggested that since the implementation of the national neonatal

216 HBV vaccination program, the overall chronic HBV carrier rate has significantly decreased and

217 has consistently remained $<1 \%$ in the student population after the birth year 1992 . In addition, a 
218 significant decrease was observed in the anti-HBs prevalence rates in the cohorts after the

219 implementation of the vaccination program. An additional decline in the prevalence of anti-HBs

220 positivity was observed when the national plasma-derived vaccine scheme was replaced by the

221 recombinant vaccine scheme in 1992. The national HBV vaccination program was a major strategy

222 implemented for the prevention of HBV infection over the years. Our study, which is one of the

223 first to use an APC model to assess the efficacy of the national HBV vaccination program,

224 suggested that the program has been effectively preventing HBV infection since its

225 commencement.

226 In our study, after the implementation of the national HBV vaccination program in 1984, the

227 prevalence rate of chronic HBV infection declined from 4.2\% (males: $4.3 \%$ vs females: $4.2 ; P=$

2280.899 ) to approximately $0.6 \%$ (males: $0.6 \%$ vs females: $0.6 \% ; P=0.889$ ) in the university students

229 born in 1999. Furthermore, according to our 2008 study, the prevalence rates of positive serum

230 anti-HBc in the students in birth cohort years 1976, 1984, and 1986, were 48.7\%, 9.8\%, and 4.0\%,

231 respectively, and the total prevalence rate of anti-HBc positivity among the students aged 18-20

232 years was 4.3\% (Su et al. 2008). Because the presence of anti-HBc indicates previous or ongoing

233 infection with HBV, in this study, we also included the serum anti-HBc titer data of students aged

$23418-20$ years in 2012 and 2016, the mean values of which were $1.7 \%$ and $0.6 \%$, respectively. These

235 observations suggest that the implementation of $\mathrm{HBV}$ vaccination program reduced the prevalence 
236 of natural HBV infection, which is consistent with the findings of previous studies. A 2017

237 Taiwanese study demonstrated that the prevalence rates of chronic HBV infection in the plasma-

238 derived and recombinant vaccination groups were 1.5\% and $0.3 \%$, respectively, in 38,000

239 university students recruited from 2003 to 2015 (Hsu et al. 2017). In addition, Ni et al. reported

240 that 25 years after the introduction of the universal neonatal HBV vaccination program in Taiwan,

241 the seroprevalence of HBV decreased from 9.8\% (prevaccination period) to <1\% (Ni et al. 2012).

$242 \mathrm{Ni}$ et al. also noted that among $3299 \mathrm{HBV}$-vaccinated young people, only $0.5 \%$ tested positive for

243 HBV infection 30 years after the national neonatal HBV vaccination program (Ni et al. 2016). This

244 suggested that among the younger students who were born after the implementation of the HBV

245 vaccination program in 1984, the prevalence rate of chronic HBV infection decreased to $<1 \%$.

246 Taiwan has already transformed from a hyper- to a low-endemic region for HBV infection (Ni et

247 al. 2016).

248 Results from our previous studies and the current study have also suggested a strong

249 association between changes in the policies of the national HBV vaccination program and the HBV

250 immunity status of our students in different birth cohort years. The prevalence of chronic HBV

251 infection decreased significantly after the implementation of the national HBV vaccination

252 program in July 1984. We also observed a decline in the anti-HBs positivity among the birth year

253 groups of July 1984 to June 1986, July 1986 to June 1992, and after July 1992, which represent 
254 the periods in which the plasma-derived HBV vaccination was administered to high-risk infants,

255 plasma-derived vaccination was administered to all infants, and recombinant vaccination was

256 administered to all infants, respectively. The stepwise decreasing trend in HBV immunity (anti-

257 HBs positivity) shown in Figure 2 can also be explained using the time frame of national HBV

258 vaccination policy change. The persistence of HBV immunity (anti-HBs positivity) in students

259 born before 1984 is primarily due to the high percentage of HBV immunity caused by natural

260 infection. With the implementation of the HBV vaccination program in July 1984, passive

261 immunity due to HBV vaccination (HBsAg negative, anti-HBs positive, and anti-HBc negative)

262 has become the predominant constitution of the HBV immunity. Some previous studies have

263 reported higher disappearance rates of anti-HBs after recombinant HBV vaccination than after

264 plasma-derived vaccination within 12-15 years after primary vaccination (Floreani et al. 2004;

265 Kao et al. 2009). Consequently, the prevalence of HBV immunity among young students born

266 after 1992 decreased sharply when the recombinant HBV vaccine was introduced in November

267 1992. Moreover, in our study, the 1992-1994 birth year was defined from July 1, 1992, to June

268 30, 1994. The administration of the recombinant three-dose yeast-derived vaccine commenced

269 from November 1, 1992. Hence, the students who were born between July 1, 1992, and October

27030,1992 , received the four-dose plasma-derived vaccine; this may satisfactorily explain why the

271 mean anti-HBs antibody level in the 1992-1994 group (which received plasma-derived and 
272 recombinant HBV vaccines) was higher than the mean levels in the 1994-1996 and 1996-1999

273 groups (which received only recombinant $\mathrm{HBV}$ vaccine).

274 Hence, our observation suggests that the prevalence rates of HBV immunity (anti-HBs

275 positivity) in fact continuously declined from the group that acquired it from anti-HBs natural-

276 boosting and the group that acquired it from plasma-derived HBV vaccines, to the group that

277 acquired anti-HBs positivity from the recombinant vaccine. By contrast, Yuen et al. reported a

278 nonsignificant difference between plasma-derived and recombinant vaccines in children aged $<11$

279 years (Yuen et al. 1999). However, in a recent study, Lin et al. reported that students in the plasma-

280 derived group exhibited a higher persistence rate of anti-HBs positivity (43.2\%) than did their

281 recombinant counterparts (33.3\%) ( $\mathrm{Li}$ et al. 2015). They also suggested that the difference in

282 antigen content between the neonatal plasma-derived and recombinant vaccines might have

283 influenced the antigen persistence and immune memory (Lin et al. 2011). Samandari et al.

284 conducted a study in Alaska, which reported that 11- to 14-year-old adolescents who had received

285 the plasma-derived vaccine at birth had 7\% higher rates of anti-HBs positivity than their

286 counterparts who had received the recombinant vaccine (14\%) in the same age group (Samandari

287 et al. 2007). Kao et al. also observed that among adolescents aged 13-15 years, anti-HBs positivity

288 was exhibited by $64.5 \%$ of the group that had received the plasma-derived vaccination (plasma-

289 derived group) born to mothers with chronic HBV infection between July 1984 and June 1986, 
$29044.1 \%$ of the plasma-derived group born between July 1986 and June 1992, and 36.0\% of the

291 recombinant group born between July 1992 and June $1995(P<.001)$ (Kao et al. 2009). In a more

292 recent study, Hsu et al. reported that the seroprevalence of HBV immunity in the recombinant

293 group was 39\% lower than that in the plasma-derived group (Hsu et al. 2017). However, both types

294 of vaccines have been reported to be comparable in terms of long-term immunogenicity and

295 protective efficacy in many studies conducted in different populations (Dentinger et al. 2005; Kao

296 et al. 2009). The persistence of serum anti-HBs level is likely affected by the types of vaccines,

297 brands, doses, and times of administration of the primary HBV vaccination as well as by high re-

298 exposure rates to HBV (Hsu et al. 2017; Yuen et al. 1999).

299 In this study, sex disparity was observed in chronic HBV infection, particularly in the

300 prenational HBV vaccination era, and in the reduced immune response to the HBV vaccine.

301 Several factors have been reported to adversely affect the antibody response to HBsAg including

302 the site and route of injection, sex, advanced age, body mass (being overweight), nutritional status,

303 smoking, and genetic factors (Chang 2006; Morris et al. 1989; Zuckerman 2006). In one previous

304 Taiwanese study, chronic HBV carrier prevalence rate was also observed to be higher in male than

305 in female students $(10.7 \%$ vs $4.4 \%)$ who born prior to the introduction of national neonatal HBV

306 vaccination and were followed up for $>18$ years in Taiwan (Su et al. 2007a). In another Taiwanese

307 case-control study, after adjusting for confounding factors, the elevated baseline of serum HBV 
308 titer was found to be significantly associated with male HBV carriers (Chen et al. 2009).

309 By setting HBsAg as the random variable for constructing the APC model, we could

310 investigate any significant difference in the prevalence under different variables of time. The

311 results revealed that the prevalence rates of chronic HBV infection in our student population

312 exhibited significant age, period, and cohort effects $(P<0.001)$. A recent population-based survey

313 and longitudinal follow-up study conducted in Taiwan demonstrated that the seromarkers of HBV

314 infection generally increase with age in the pre- and postvaccination generations (Chen et al. 2015).

315 Multiple studies have discussed the period and cohort effects in detail and have reported a

316 significant decrease in HBV seromarkers in the cohorts after the implementation of the vaccination

317 program (Ni et al. 2016; Ni et al. 2012; Ni et al. 2007). Small-scale clinical trials have also

318 consistently established that neonatal vaccinations can effectively prevent acute and chronic HBV

319 infection (Bruce et al. 2016; Dumaidi \& Al-Jawabreh 2015; Huang et al. 2015; Su et al. 2013).

320 The significant cohort effect further confirms that HBV disease prevalence decreases with each

321 generation. Previous seroepidemiological studies in Taiwan have demonstrated an HBV

322 vaccination coverage rate ( $\geq 3$ doses of vaccine) of $89.5 \%$ in Taiwan in $1986,92.8 \%$ before

323 December 2002, and 97\% in the 2002 birth cohort (Gust 1996; Lin et al. 2011; Ni et al. 2007). The

324 vaccination coverage rate has already reached its peak plateau and is likely among the highest

325 globally. The implementation of the national HBV vaccination program in 1984 was a major 
326 intervention tool for combating HBV infection, and our APC model demonstrated that the program

327 has been highly successful in preventing HBV infection in young adults.

328 With the implementation of national HBV vaccination and the accompanying measures in

329 public health and preventive medicine, the majority of $\mathrm{HBV}$ acquisition in the post-HBV

330 vaccination era has been successfully controlled. A recent study demonstrated that approximately

$33180 \%$ of the HBV carriers born in the post-HBV vaccination era had a positive maternal status, and

332 the authors suggest that mother-infant vertical transmission remains a crucial cause of vaccine

333 failure (Ni et al. 2016). Additional prenatal precautionary measures should be considered in

334 addition to the routine implementation of the HBV vaccination program for total HBV eradication.

335 Some limitations of this study must be discussed. Because this data set was delinked, the

336 vaccination history of the participants and the carrier status of the participants' mothers were not

337 available. However, our HBV carrier and anti-HBs positivity rates were comparable with those

338 reported in previous studies. Our HBV vaccine coverage rate can be assumed to be similar to that

339 of other studies in Taiwan because all the university new entrants were under the same national

340 HBV scheme. Second, the data set was collected from a single university in northern Taiwan.

341 However, the university admission was based on national university and graduate school entrance

342 examination results. Consequently, the students were from various parts of Taiwan, which

343 minimized the selection bias. Third, a slight chance of duplicate data may exist because some 
344 students may possess health records at both college and graduate levels in the same school.

345 However, this selection bias was minimized through a careful cross verification prior to the

346 identification delinking process. Finally, we did not provide the annual anti-HBc data representing

347 the status of HBV natural infection. However, by using the data of our previous study in 2008 and

348 the data of anti-HBc status in 2012 and 2016, we presented a general declining trend in anti-HBc

349 positivity in our study.

350

351 Conclusion:

352

This study found that the prevalence rate of chronic HBV infection decreased to $<1 \% 30$

353 years after the implementation of national HBV vaccination in 1984. Our APC model revealed

354 that HBV vaccination is one of the most effective strategies for preventing HBV infection. The

355 HBV vaccination program has transformed Taiwan from a hyper- to a low-endemic region.

356 However, the complete eradication of HBV infection requires additional effective strategies for

357 detecting vaccination failure and the early implementation of appropriate treatments. Further

358 research is warranted for investigating these strategies. 


\section{References}

361 Beasley RP, and Hwang LY. 1984. Hepatocellular carcinoma and hepatitis B virus. Semin Liver 362 Dis 4:113-121. 10.1055/s-2008-1040651

363 364 365 366

367

368

369

370

371

372

373

374

375

376

377

378

379

380

381

382

383

384

385

386

387

388

389

390

391

392

393

394

Bruce MG, Bruden D, Hurlburt D, Zanis C, Thompson G, Rea L, Toomey M, Townshend-Bulson L, Rudolph K, Bulkow L, Spradling PR, Baum R, Hennessy T, and McMahon BJ. 2016. Antibody Levels and Protection After Hepatitis B Vaccine: Results of a 30-Year Followup Study and Response to a Booster Dose. J Infect Dis 214:16-22. 10.1093/infdis/jiv748

Chang MH. 2006. Impact of hepatitis B vaccination on hepatitis B disease and nucleic acid testing in high-prevalence populations. J Clin Virol 36 Suppl 1:S45-50.

Chen CJ, Yang HI, Iloeje UH, and Group R-HS. 2009. Hepatitis B virus DNA levels and outcomes in chronic hepatitis B. Hepatology 49:S72-84. 10.1002/hep.22884

Chen CL, Yang JY, Lin SF, Sun CA, Bai CH, You SL, Chen CJ, Kao JH, Chen PJ, and Chen DS. 2015. Slow decline of hepatitis B burden in general population: Results from a populationbased survey and longitudinal follow-up study in Taiwan. J Hepatol 63:354-363. 10.1016/j.jhep.2015.03.013

Chen DS, Hsu NH, Sung JL, Hsu TC, Hsu ST, Kuo YT, Lo KJ, and Shih YT. 1987. A mass vaccination program in Taiwan against hepatitis $\mathrm{B}$ virus infection in infants of hepatitis $\mathrm{B}$ surface antigen-carrier mothers. JAMA 257:2597-2603.

Chien YC, Jan CF, Kuo HS, and Chen CJ. 2006. Nationwide hepatitis B vaccination program in Taiwan: effectiveness in the 20 years after it was launched. Epidemiol Rev 28:126-135. 10.1093/epirev/mxj010

Center for Disease Control, Taiwan. 2017. 2015 National Various Immunization Completion Rate. http://www.cdc.gov.tw/professional/page.aspx?treeid=5b0231beb94edffc\&nowtreeid=3D 1CDC342B28E123. Accessed April 15, 2017.

Dentinger CM, McMahon BJ, Butler JC, Dunaway CE, Zanis CL, Bulkow LR, Bruden DL, Nainan OV, Khristova ML, Hennessy TW, and Parkinson AJ. 2005. Persistence of antibody to hepatitis B and protection from disease among Alaska natives immunized at birth. Pediatr Infect Dis J 24:786-792.

Dumaidi K, and Al-Jawabreh A. 2015. Persistence of Anti-HBs Among Palestinian Medical Students After 18 - 22 Years of Vaccination: A Cross-Sectional Study. Hepat Mon 15:e29325. 10.5812/hepatmon.29325

Floreani A, Baldo V, Cristofoletti M, Renzulli G, Valeri A, Zanetti C, and Trivello R. 2004. Longterm persistence of anti-HBs after vaccination against HBV: an 18 year experience in health care workers. Vaccine 22:607-610. 
395 GmbH BM. 1998. Elecsys 2010 System: Elecsys HBsAg Immunoassay and Elecsys HBsAg

396

397

398

399

400

401

402

403

404

405

406

407

408

409

410

411

412

413

414

415

416

417

418

419

420

421

422

423

424

425

426

427

428

429

430 Confirmatory Test. Boehringer Mannheim GmbH Elecsys 2010 System Product Information

GmbH RD. 2007a. Elecsys 2010 anti-HBc assay: Antibodies to hepatitis B core antigen.

GmbH RD. 2007b. Elecsys 2010 anti-HBs assay: Antibody to hepatitis B surface antigen.

Gust ID. 1996. Immunisation against hepatitis B in Taiwan. Gut 38 Suppl 2:S67-68.

Hsu SH, Chih AH, Lee YC, Huang KC, and Jan CF. 2017. Higher disappearance rate of anti-HBs in Taiwanese freshers neonatally vaccinated with recombinant yeast hepatitis B vaccine. Liver Int 37:1780-1787. 10.1111/liv.13437

Huang P, Zhu LG, Zhu YF, Yue M, Su J, Zhu FC, Yang HT, Zhang Y, Shen HB, Yu RB, Zhai XJ, and Peng ZH. 2015. Seroepidemiology of hepatitis B virus infection and impact of vaccination. World J Gastroenterol 21:7842-7850. 10.3748/wjg.v21.i25.7842

Kao JT, Wang JH, Hung CH, Yen YH, Hung SF, Hu TH, Lee CM, and Lu SN. 2009. Long-term efficacy of plasma-derived and recombinant hepatitis B vaccines in a rural township of Central Taiwan. Vaccine 27:1858-1862. 10.1016/j.vaccine.2009.01.027

Li HJ, Zhai NC, Song HX, Yang Y, Cui A, Li TY, and Tu ZK. 2015. The Role of Immune Cells in Chronic HBV Infection. J Clin Transl Hepatol 3:277-283. 10.14218/JCTH.2015.00026

Lin CC, Yang CY, Shih CT, Chen BH, and Huang YL. 2011. Waning immunity and booster responses in nursing and medical technology students who had received plasma-derived or recombinant hepatitis B vaccine during infancy. Am J Infect Control 39:408-414. 10.1016/j.ajic.2010.07.010

Morris CA, Oliver PR, Reynolds F, and Selkon JB. 1989. Intradermal hepatitis B immunization with yeast-derived vaccine: serological response by sex and age. Epidemiol Infect 103:387394.

Ni YH, Chang MH, Huang LM, Chen HL, Hsu HY, Chiu TY, Tsai KS, and Chen DS. 2001. Hepatitis B virus infection in children and adolescents in a hyperendemic area: 15 years after mass hepatitis B vaccination. Ann Intern Med 135:796-800.

Ni YH, Chang MH, Jan CF, Hsu HY, Chen HL, Wu JF, and Chen DS. 2016. Continuing Decrease in Hepatitis B Virus Infection 30 Years After Initiation of Infant Vaccination Program in Taiwan. Clin Gastroenterol Hepatol 14:1324-1330. 10.1016/j.cgh.2016.04.030

Ni YH, Chang MH, Wu JF, Hsu HY, Chen HL, and Chen DS. 2012. Minimization of hepatitis B infection by a 25-year universal vaccination program. $J$ Hepatol 57:730-735. 10.1016/j.jhep.2012.05.021

Ni YH, Huang LM, Chang MH, Yen CJ, Lu CY, You SL, Kao JH, Lin YC, Chen HL, Hsu HY, and Chen DS. 2007. Two decades of universal hepatitis B vaccination in taiwan: impact and implication for future strategies. Gastroenterology 132:1287-1293. 
431

432

433

434

435

436

437

438

439

440

441

442

443

444

445

446

447

448

449

450

451

452

453

454

455

456

457

458

459

460

461

462

463

464

465

466

10.1053/j.gastro.2007.02.055

Perz JF, Armstrong GL, Farrington LA, Hutin YJ, and Bell BP. 2006. The contributions of hepatitis $\mathrm{B}$ virus and hepatitis $\mathrm{C}$ virus infections to cirrhosis and primary liver cancer worldwide. J Hepatol 45:529-538. 10.1016/j.jhep.2006.05.013

Samandari T, Fiore AE, Negus S, Williams JL, Kuhnert W, McMahon BJ, and Bell BP. 2007. Differences in response to a hepatitis B vaccine booster dose among Alaskan children and adolescents vaccinated during infancy. Pediatrics 120:e373-381. 10.1542/peds.2007-0131

Su FH, Chen JD, Cheng SH, Lin CH, Liu YH, and Chu FY. 2007a. Seroprevalence of Hepatitis$\mathrm{B}$ infection amongst Taiwanese university students 18 years following the commencement of a national Hepatitis-B vaccination program. $J$ Med Virol 79:138-143. 10.1002/jmv.20771

Su FH, Chen JD, Cheng SH, Sung KY, Jeng JJ, and Chu FY. 2008. Waning-off effect of serum hepatitis B surface antibody amongst Taiwanese university students: 18 years postimplementation of Taiwan's national hepatitis B vaccination programme. $J$ Viral Hepat 15:14-19. 10.1111/j.1365-2893.2007.00890.x

Su FH, Cheng SH, Li CY, Chen JD, Hsiao CY, Chien CC, Yang YC, Hung HH, and Chu FY. 2007b. Hepatitis B seroprevalence and anamnestic response amongst Taiwanese young adults with full vaccination in infancy, 20 years subsequent to national hepatitis B vaccination. Vaccine 25:8085-8090. 10.1016/j.vaccine.2007.09.013

Su FH, Chu FY, Bai CH, Lin YS, Hsueh YM, Sung FC, and Yeh CC. 2013. Efficacy of hepatitis B vaccine boosters among neonatally vaccinated university freshmen in Taiwan. $J$ Hepatol 58:684-689. 10.1016/j.jhep.2012.11.036

Sung JL. 1984. Hepatitis B virus infection and its sequelae in Taiwan. Gastroenterol Jpn 19:363366.

Tsen YJ, Chang MH, Hsu HY, Lee CY, Sung JL, and Chen DS. 1991. Seroprevalence of hepatitis B virus infection in children in Taipei, 1989: five years after a mass hepatitis B vaccination program. J Med Virol 34:96-99.

World Health Organiztion. 2017. Global hepatitis report. http://www.who.int/hepatitis/publications/global-hepatitis-report2017/en/_Accessed November 29, 2017

Yuen MF, Lim WL, Cheng CC, Lam SK, and Lai CL. 1999. Twelve-year follow-up of a prospective randomized trial of hepatitis B recombinant DNA yeast vaccine versus plasmaderived vaccine without booster doses in children. Hepatology 29:924-927. 10.1002/hep.510290327

Zuckerman JN. 2006. Protective efficacy, immunotherapeutic potential, and safety of hepatitis B vaccines. J Med Virol 78:169-177. 10.1002/jmv.20524 
467

468

469

PeerJ reviewing PDF | (2017:09:20928:1:1:NEW 24 Dec 2017) 


\section{Table $\mathbf{1}$ (on next page)}

Hepatitis B virus infection status according to birth year of students who had enrolled between 2005 and 2016 at a university in northern Taiwan 
Table 1. Hepatitis B virus infection status according to birth year of students who had enrolled between 2005 and 2016 at a university in northern Taiwan

\begin{tabular}{|c|c|c|c|c|c|c|c|c|c|c|c|}
\hline \multirow[b]{3}{*}{ Birth Year } & \multirow{3}{*}{$\begin{array}{c}\text { Tota } \\
1 \\
n\end{array}$} & \multicolumn{2}{|c|}{ Sex } & \multicolumn{4}{|c|}{ HBsAg +ve } & \multicolumn{4}{|c|}{ HBsAg -ve, Anti-HBs +ve } \\
\hline & & Female & Male & Total & Female & Male & $\begin{array}{l}P \text { value } \\
\left(\text { For } \chi^{2}\right)\end{array}$ & Total & Female & Male & $\begin{array}{l}P \text { value } \\
\left(\text { For } \chi^{2}\right)\end{array}$ \\
\hline & & $\mathrm{n}$ & $\mathrm{n}$ & $\mathrm{n}(\%)^{\dagger}$ & $\mathrm{n}(\%)$ & $\mathrm{n}(\%)^{\S}$ & & $\mathrm{n}(\%)^{\dagger}$ & $\mathrm{n}(\%)$ & $\mathrm{n}(\%)^{\S}$ & \\
\hline $1974 / 7-1979 / 6$ & 958 & 699 & 259 & $98(10.2)$ & $63(9.0)$ & $35(13.5)$ & $<0.001$ & $744(77.7)$ & $559(80.0)$ & $185(71.4)$ & $<0.001$ \\
\hline $1979 / 7-1984 / 6$ & 1633 & 1131 & 502 & $108(6.6)$ & $70(6.2)$ & $38(7.6)$ & 0.039 & $1320(80.8)$ & $945(83.6)$ & $375(74.7)$ & $<0.001$ \\
\hline 1984/7-1986/6 & 969 & 619 & 350 & $41(4.2)$ & $26(4.2)$ & $15(4.3)$ & 0.899 & $758(78.2)$ & $505(81.6)$ & $253(72.3)$ & $<0.001$ \\
\hline 1990/7-1992/6 & 2317 & 1388 & 929 & $31(1.3)$ & $15(1.1)$ & $16(1.7)$ & 0.008 & $1404(60.6)$ & $884(63.7)$ & $520(56.0)$ & $<0.001$ \\
\hline 1992/7-1994/6 & 2267 & 1373 & 894 & $16(0.7)$ & $9(0.7)$ & $7(0.8)$ & 0.479 & 1007 (44.4) & $626(45.6)$ & $381(42.6)$ & 0.004 \\
\hline 1994/7-1996/6 & 1980 & 1188 & 792 & $6(0.3)$ & $2(0.2)$ & $4(0.5)$ & 0.008 & $717(36.2)$ & $440(37.0)$ & $277(35.0)$ & 0.058 \\
\hline 1996/7-1999/6 & 1739 & 1062 & 677 & $10(0.6)$ & $6(0.6)$ & $4(0.6)$ & 0.889 & 597 (34.3) & $370(34.8)$ & $227(33.5)$ & 0.258 \\
\hline
\end{tabular}

6 : Percentage of total population in the birth year

7 : Percentage of total female population in the birth year

8 \&: Percentage of total male population in the birth year

9 T: $P$ value was used for comparing the percentages of female and male populations in a given birth year

10 Abbreviations: HBsAg, HBV surface antigen; Anti-HBs, antibody against HBsAg 


\section{Table 2 (on next page)}

Distribution of students who had enrolled between 2005 and 2016 at a university in Taiwan into different age-period-cohort groups. 
Table 2. Distribution of students who had enrolled between 2005 and 2016 at a university in Taiwan into different age-period-cohort groups.

\begin{tabular}{lccccc}
\hline & Cohort A & Cohort B & Cohort C & Cohort D & Total \\
\hline Sample size (\%) & $3842(21.8 \%)$ & $968(5.5 \%)$ & $7220(41.0 \%)$ & $5581(31.7 \%)$ & 17611 \\
Age (mean \pm SD) & $32.8 \pm 7.8$ & $23.4 \pm 3.1$ & $19.8 \pm 2.4$ & $18.9 \pm 1.0$ & $22.6 \pm 6.8$ \\
Female & $2671(69.5 \%)$ & $620(64.0 \%)$ & $4361(60.4 \%)$ & $3386(60.7 \%)$ & $11038(62.7 \%)$ \\
\hline Entry year & & & & & \\
2005 & 709 & 234 & 668 & 0 & 1611 \\
2006 & 651 & 67 & 830 & 0 & 1548 \\
2007 & 483 & 139 & 924 & 0 & 1546 \\
2008 & 326 & 163 & 950 & 0 & 1439 \\
2009 & 262 & 87 & 1002 & 0 & 1351 \\
2010 & 350 & 48 & 1036 & 6 & 1440 \\
2011 & 294 & 66 & 585 & 613 & 1558 \\
2012 & 199 & 47 & 368 & 871 & 1485 \\
2013 & 181 & 42 & 308 & 935 & 1466 \\
2014 & 127 & 30 & 209 & 958 & 1324 \\
2015 & 153 & 23 & 173 & 1106 & 1455 \\
2016 & 107 & 22 & 167 & 1092 & 1388 \\
\hline
\end{tabular}

4

5 Cohort A: Students born before July 1, 1984;

6 Cohort B: Students born between July 1, 1984, and June 30, 1986, inclusively;

7 Cohort C: Students born between July 1, 1986, and October 30, 1992, inclusively;

8 Cohort D: Students born after November 1, 1992.

9 Abbreviation: SD, standard deviation 


\section{Table 3(on next page)}

Analysis of age-period-cohort effects of hepatitis B surface antigen positivity according to birth year in students who had enrolled between 2005 and 2016 from a university in Taiwan. 
1 Table 3. Analysis of age-period-cohort effects of hepatitis B surface antigen positivity according to birth year in students who had enrolled between 2005 and 2016 from a university in Taiwan.

\begin{tabular}{|c|c|c|c|c|c|c|c|}
\hline Model & $\begin{array}{l}\text { Degrees of } \\
\text { freedom }\end{array}$ & Deviance & $\begin{array}{l}\text { Likelihood } \\
\text { ratio test }\end{array}$ & Effects & $\begin{array}{l}\text { Deviance } \\
\text { difference }\end{array}$ & $\begin{array}{l}\text { df } \\
\text { difference }\end{array}$ & $\begin{array}{l}\text { P- value } \\
\left(\text { For } X^{2}\right)\end{array}$ \\
\hline Age & 15 & 620.3 & 31301.6 & & 492.7 & 9 & $<0.001$ \\
\hline Period & 16 & 334.8 & 31755.2 & & 207.1 & 10 & 0.050 \\
\hline Cohort & 12 & 415.4 & 31438.3 & & 287.7 & 6 & $<0.001$ \\
\hline Age + period & 12 & 326.4 & 458.9 & Cohort & 198.7 & 6 & $<0.001$ \\
\hline Age + cohort & 8 & 347.3 & 275.5 & Period & 219.6 & 2 & $<0.001$ \\
\hline Period + cohort & 9 & 320.3 & 323.6 & Age & 192.6 & 3 & $<0.001$ \\
\hline Age + period + cohort & 6 & 127.7 & 255.8 & & Reference & Reference & \\
\hline
\end{tabular}

16 Abbreviation: df, degrees of freedom 


\section{Figure 1}

Hepatitis B surface antigen positivity status of the students from a university in Taiwan who were enrolled between 2005 and 2016 according to birth year.

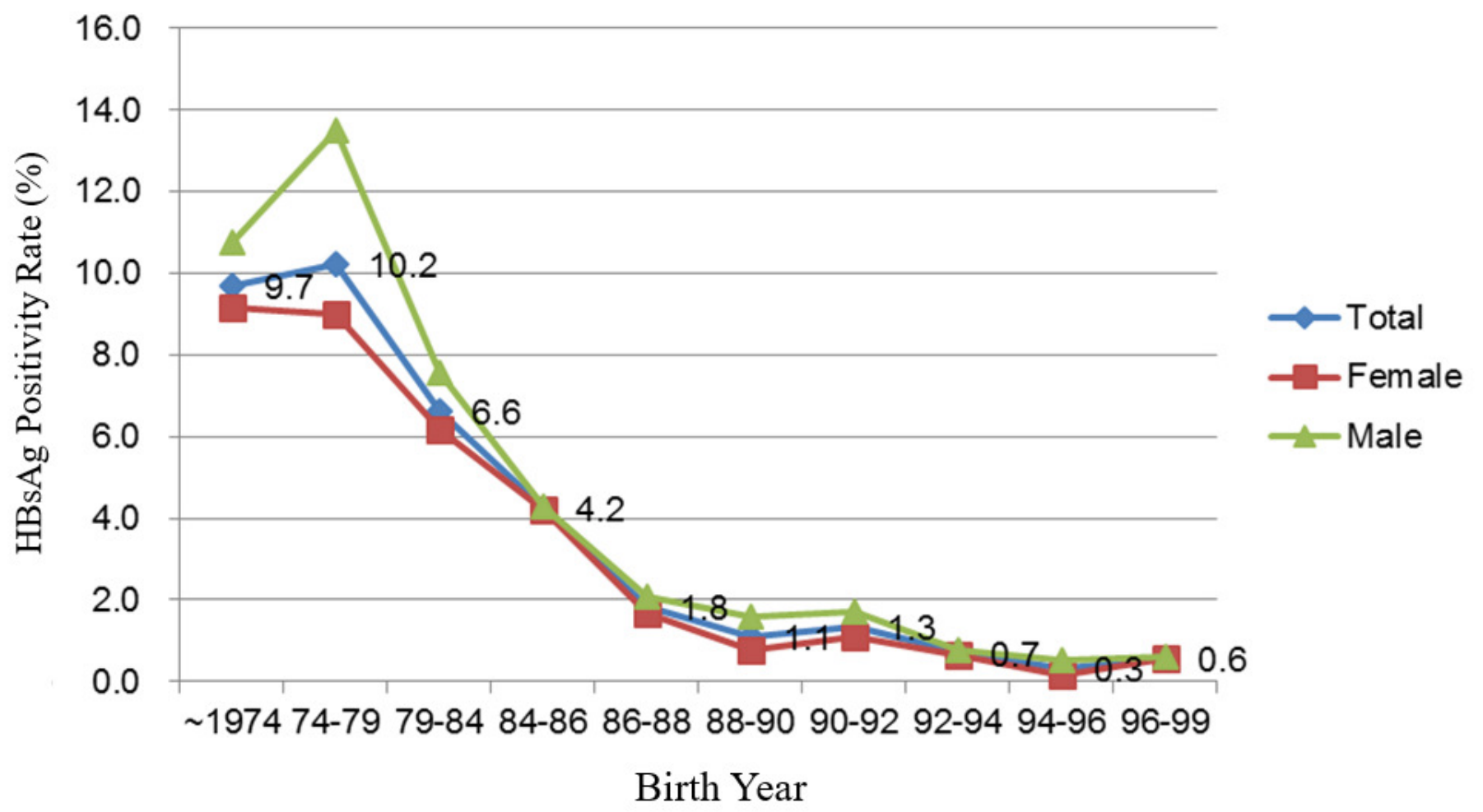


Figure 2

Antibody against hepatitis B surface antigen positivity status of the students from a university in Taiwan who were enrolled between 2005 and 2016 according to birth year.

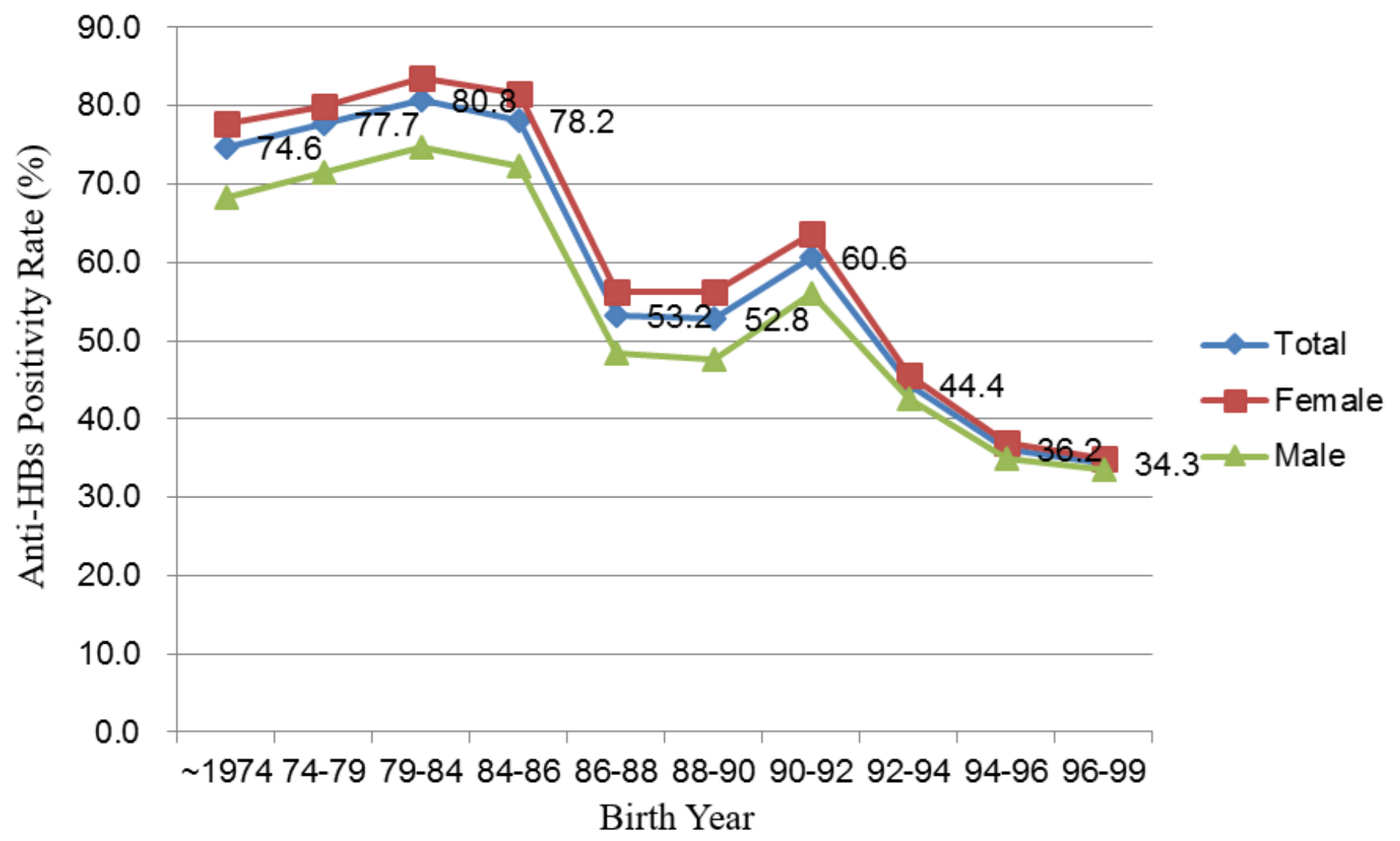

